\title{
Códigos Turbo M-ários com Modulação M-PSK
}

\author{
José da Silva Barros e Renato Baldini Filho
}

\begin{abstract}
Resumo-O objetivo deste trabalho é apresentar classes de códigos turbo M-ários. O esquema de codificação turbo consiste em dois codificadores componentes multiníveis, concatenados em paralelo via um entrelaçador, mapeados através da constelação M-PSK (M-ary Phase Shift Keying) e transmitidos pelo canal de comunicação AWGN (Aditive White Gaussian Noise). O esquema de decodificação turbo é formado por um algoritmo de decodificação M-ário, baseado na decodificação MAP (máximo a posteriori) via a decodificação iterativa. Os resultados das simulações mostram que os códigos turbo M-ário são mais eficientes que os códigos turbo binários.
\end{abstract}

Palavras-Chave-Códigos Turbo M-ário, Algoritmo de Decodificação Iterativa.

Abstract-The aim of this work is to present classes of M-ary turbo codes. The encoding scheme consists of two M-ary systematic recursive convolutional encoders separated by s-random interleaver. The decoding scheme is based on the maximum $a$ posteriori decoding algorithm - MAP. Simulation results show that $M$-ary turbo codes are more efficient than the binary turbo codes.

Keywords - M-ary Turbo Codes, Iterative Decoding Algorithm.

\section{INTRODUÇÃO}

Desde que Ungerboeck introduziu os conceitos de códigos treliça, em 1982 [3], os códigos turbo binários apresentados por C. Berrou, A. Glavieux e P. Thitimajashima em 1993 [1], representam a mais importante descoberta na área de teoria da informação e codificação, por apresentar desempenho muito próximo ao limitante de Shannon para o canal $A W G N$ (Aditive White Gaussian Noise). A estrutura entrelaçada dos códigos permite uma decodificação próxima a decodificação de máximo a posteriori (MAP) via decodificação iterativa.

A maioria dos códigos turbo pesquisados consideram códigos componentes binários concatenados via um entrelaçador aleatório de $N$ bits [1], [2], [4] e [5]. Neste artigo, consideramos os códigos turbo construídos para códigos componentes não binários (ou M-ários), concatenados via um entrelaçador aleatório de $N$ símbolos e mapeado em uma constelação apropriada $M-P S K$, para transmissão através de um canal $A W G N$. A decodificação usada para investigar o desempenho dos códigos turbo $M$-ários é a decodificação de máximo a posteriori também via decodificação iterativa.

O casamento natural do tamanho da constelação com o tamanho do alfabeto, dá maior flexibilidade a combinação: complexidade de decodificação, capacidade de correção de erro, cardinalidade do alfabeto, largura de banda e mapeamento de bits para símbolos quando analisamos o desempenho do esquema turbo multinível. Assim, os esquemas

José da Silva Barros e Prof. Dr. Renato Baldini Filho, Faculdade de Engenharia Elétrica e de Computação, Departamento de Telecomunicações, UNICAMP, Campinas, Brasil, E-mails: barros@decom.fee.unicamp.br, baldini@decom.fee.unicamp.br. Este trabalho foi financiado pela FAPEAL(E.20031029439-0). turbo $M$-ários se apresentam mais eficientes, com relação aos parâmetros mencionados acima, do que os esquemas turbo binários.

\section{CodificaÇÃo Turbo $M$-ária}

$\mathrm{O}$ esquema de codificação turbo $M$-ário é constituído por dois codificadores convolucionais geralmente idênticos, de taxa $1 / 2$, separados por um entrelaçador de $N$ símbolos, juntamente com um mecanismo opcional de puncionamento. Esses dois codificadores constituintes são sistemáticos recursivos e utilizados em paralelo, conforme a Figura 1.

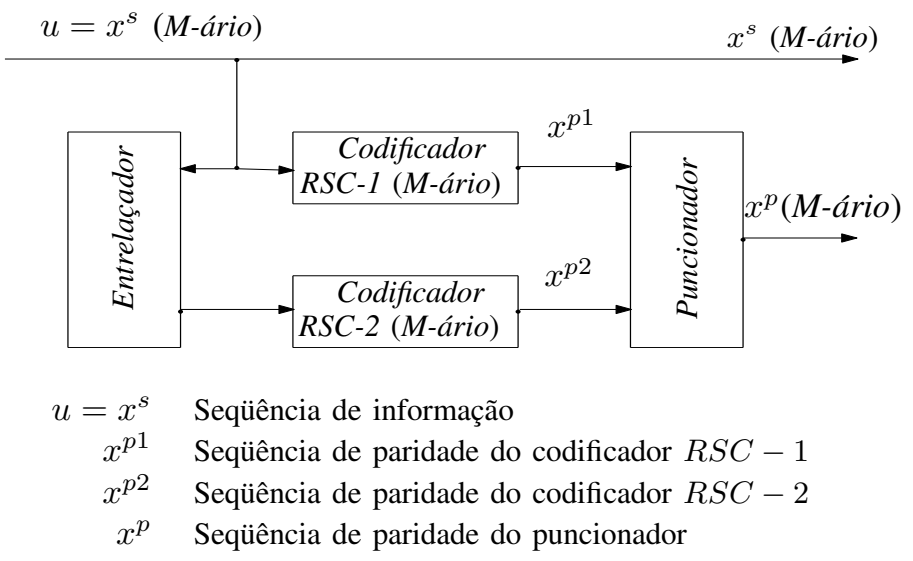

Fig. 1. Esquema de codificação turbo.

\section{A. Codificador Convolucional Sistemático Recursivo}

Seja $\mathbb{Z}_{M}$ um anel de inteiros comutativo com identidade multiplicativa e seja $L_{r}(D)$ o anel formado por polinômios racionais do tipo $q(D) / p(D)$, onde $q(D)$ e $p(D)$ são polinômios com coeficientes em $\mathbb{Z}_{M}$, expressos pela série de Laurent [6], [7] e [9], $f(D)=\sum_{i=0}^{n} f_{i} D^{i}, \operatorname{com} f_{i} \in \mathbb{Z}_{M}$ e $p(0) \neq 0$ em $\mathbb{Z}_{M}$ - chamado anel das funções realizáveis sobre $\mathbb{Z}_{M}$.

Assim, um codificador convolucional sistemático recursivo RSC (Recursive Systematic Convolutional), com taxa de codificação $k / n$ sobre o anel de funções realizáveis $L_{r}(D)$, é um mapeamento linear expresso como

$$
v(D)=u(D) G_{r}(D),
$$

onde $G_{r}(D)$ é uma matriz $k \times n$ na forma sistemática

$$
G_{r}(D)=\left[\begin{array}{ll}
I_{k} & X(D)
\end{array}\right],
$$

e $u(D)$ é o polinômio correspondente a seqüência de informação. Observe que $I_{k}$ é uma matriz identidade $k \times k$ e $X(D)$ é uma matriz $k \times(n-k)$ com elementos cuja representação é $q(D) / p(D)$ em $L_{r}(D)$.

Portanto, o conjunto

$$
C=\left\{u(D) g(D) \mid u(D) \in L_{r}(D)^{k}\right\},
$$


é um código $R S C$ com taxa de codificação $k / n$ sobre $\mathbb{Z}_{M}$, onde $g(D)=G_{r}(D)$ é a matriz geradora com elementos em $L_{r}(D)$.

\section{B. Entrelaçador}

Os entrelaçadores, usados com grande frequiência nos meios de comunicação, têm por objetivo espalhar erros que ocorrem em surtos (burst), causados pelo ruído impulsivo e pelo desvanecimento (fading). Usamos em nossa simulações o entrelaçador s-aleatório [8], pois este apresenta melhores propriedades aleatórias.

\section{Puncionador}

A função do puncionador é apagar periodicamente símbolos de redundância pré-selecionados dos codificadores, aumentando a taxa de codificação. Quando usamos o puncionamento, são mapeados $N$ símbolos de informação em $2 N$ símbolos do código, enquanto que, sem o puncionamento, são mapeados $N$ símbolos de informação em $3 N$ símbolos do código.

\section{Modulador M-PSK}

A sequiência de entrada do modulador é composta por símbolos pertencentes ao anel de inteiros $\mathbb{Z}_{M}$. Estes símbolos são transformados através da modulação $M-P S K$ em sinais do tipo

$$
S_{i}=A \exp \left(j \frac{2 \pi i}{M}\right),
$$

onde $i$ é um símbolo do alfabeto $M$-ário, $\{0,1, \cdots, M-1\} \in$ $\mathbb{Z}_{M}$ e $A$ é uma amplitude (raio) do sinal.

A relação fechada que existe entre o anel de inteiros módulo- $M$ e a modulação $M-P S K$, justifica o ganho no desempenho do sistema turbo, referente ao perfeito casamento dos símbolos do alfabeto $M$-ário com a modulação $M$-PSK.

\section{Algoritmo de DecodificaÇÃo}

A função do algoritmo MAP é fornecer a informação $a$ posteriori $L\left(u_{k}\right)$ na saída de cada decodificador, definida da seguinte maneira. Seja

$$
L\left(u_{k}\right)=\max \left(\ln \left(p\left(u_{k}=\theta \mid y\right)\right)\right),
$$

o valor máximo do logaritmo natural da probabilidade a posteriori de um símbolo decodificado ser $u_{k}=\theta \in\{0,1,2, \ldots, M-1\}$, dado que a sequiência de símbolos recebida é $y=\left(\begin{array}{llllllll}y_{1} & y_{2} & \cdots & y_{k-1} & y_{k} & y_{k+1} & \cdots & y_{N}\end{array}\right)$. Note que $M$ é o comprimento do alfabeto de entrada e $N$ é o comprimento da seqüência de informação.

Se as transições entre o estado prévio, $S_{k-1}=s^{\prime}$, e o estado presente, $S_{k}=s$, são mutuamente exclusivas (isto é, apenas uma delas pode ter ocorrido na treliça referente ao codificador RSC), então usando a regra de Bayes

$$
L\left(u_{k}\right)=\max \left(\ln \left(\frac{\sum_{\left(s^{\prime}, s\right)} p\left(S_{k-1}=s^{\prime}, S_{k}=s, y\right)}{p(y)}\right)\right),
$$

onde $\left(s^{\prime}, s\right)$ é o conjunto de transições do estado prévio, $S_{k-1}=s^{\prime}$, para o estado presente, $S_{k}=s$, que pode ocorrer se o símbolo de entrada $u_{k}$ for igual a $\theta$.

Como o canal é sem memória e os símbolos da seqüência recebida $y$ são independentes entre si, substituindo $p(y)$ por $p(y) / p\left(y_{k}\right)$ na expressão (2) temos:

$$
L\left(u_{k}\right)=\max \left(\ln \left(\sum_{\left(s^{\prime}, s\right)} \alpha_{k-1}\left(s^{\prime}\right) \cdot \gamma_{k}\left(s^{\prime}, s\right) \cdot \beta_{k}(s)\right)\right),
$$

onde $\alpha_{k}(s)$ e $\beta_{k}(s)$ são as probabilidades definidas respectivamente como:

$$
\begin{aligned}
\alpha_{k}(s) & =\frac{\sum_{\text {todo } s^{\prime}} \alpha_{k-1}\left(s^{\prime}\right) \cdot \gamma_{k}\left(s^{\prime}, s\right)}{\sum_{\text {todo } s \text { todo } s^{\prime}} \alpha_{k-1}\left(s^{\prime}\right) \cdot \gamma_{k}\left(s^{\prime}, s\right)} \mathrm{e} \\
\beta_{k-1}(s) & =\frac{\sum_{\text {todo } s} \beta_{k}(s) \cdot \gamma_{k}\left(s^{\prime}, s\right)}{\sum_{\text {todo s todo } s^{\prime}} \alpha_{k-1}\left(s^{\prime}\right) \cdot \gamma_{k}\left(s^{\prime}, s\right)} .
\end{aligned}
$$

Observe que $\alpha_{k}(s)$ e $\beta_{k}(s)$ são calculados recursivamente pelas equações (4) e (5) e possuem as condições iniciais.

$$
\alpha_{0}\left(S_{0}=s\right)=\beta_{N}\left(S_{N}=s\right)=\left\{\begin{array}{lll}
1 & \text { para } & s=0 \\
0 & \text { para } & s \neq 0 .
\end{array}\right.
$$

Com base nas mesmas hipóteses temos que a probabilidade de transição é dada por

$$
\gamma_{k}\left(s^{\prime}, s\right)=p\left(y_{k} \mid x_{k}\right) \cdot p\left(u_{k}\right) .
$$

Assim, apresentamos as expressões de $\alpha_{k-1}\left(s^{\prime}\right), \beta_{k}(s)$ e $\gamma_{k}\left(s^{\prime}, s\right)$, necessárias para calcular a informação a posteriori $L\left(u_{k}\right)$, na saída de cada decodificador componente.

\section{DecodificaÇão Iterativa Turbo $M$-ária}

O processo de decodificação iterativa turbo definido sobre o anel $\mathbb{Z}_{M}$, esquematizado na Figura 2, calcula a informação extrínseca na saída do estágio de decodificação anterior, e a usa como informação a priori na entrada do próximo estágio de decodificação.

Sabendo que cada codificador $R S C$ do esquema de codificação turbo tem taxa de codificação $1 / 2$, a palavra código transmitida é dada por $x_{k}=x_{k}^{s} x_{k}^{p}=u_{k} x_{k}^{p}$, e a palavra código recebida é dada por $y_{k}=y_{k}^{s} y_{k}^{p}$.

Assim, se o canal é sem memória, gaussiano e com modulação $M-P S K$, então, a função densidade de probabilidade é dada por

$$
\begin{aligned}
p\left(y_{k} \mid x_{k}\right) & =\frac{1}{\pi N_{0}} \cdot \exp \left(\frac{-\left\|y_{k}^{s}-x_{k}^{s}\right\|^{2}-\left\|y_{k}^{p}-x_{k}^{p}\right\|^{2}}{N_{0}}\right) \\
& =\exp \left(\frac{-\left\|y_{k}^{s}-x_{k}^{s}\right\|^{2}}{N_{0}}\right) \cdot \gamma_{k}^{e}\left(s^{\prime}, s\right),
\end{aligned}
$$

onde: $x_{k}^{s}$ é o símbolo sistemático da palavra código transmitida $x_{k} ; x_{k}^{p}$ é o símbolo de paridade da palavra código transmitida $x_{k} ; y_{k}^{s}$ é o sinal recebido correspondente ao símbolo transmitido $x_{k}^{s}$ e $y_{k}^{p}$ é o sinal recebido correspondente ao símbolo transmitido $x_{k}^{p}$. 


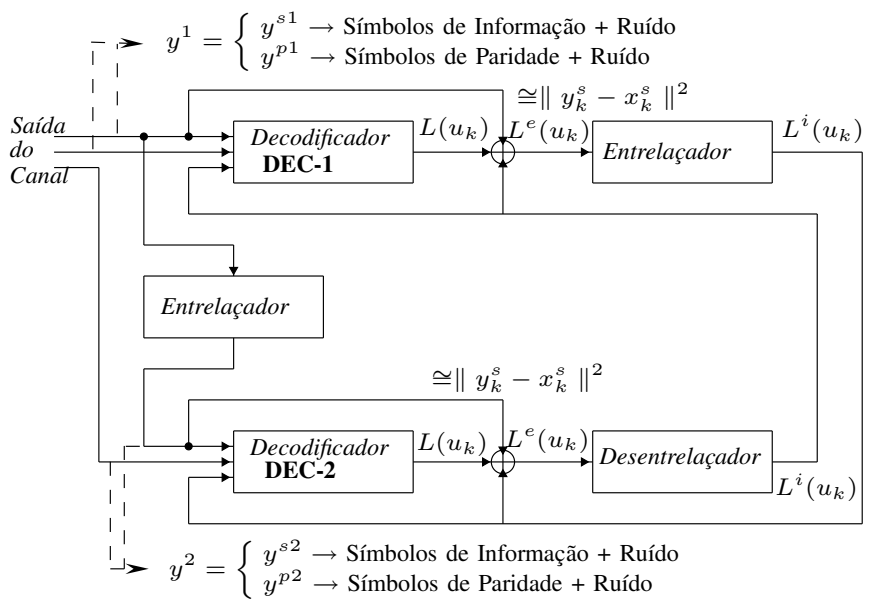

Fig. 2. Decodificação Iterativa Turbo.

Portanto, a informação a posteriori, $L\left(u_{k}\right)$, calculada através do algoritmo $M A P$ na saída de cada decodificador componente, pode ser dividida em três termos

$$
L\left(u_{k}\right)=L^{i}\left(u_{k}\right)+\exp \left(\frac{-\left\|y_{k}^{s}-x_{k}^{s}\right\|^{2}}{N_{0}}\right)+L^{e}\left(u_{k}\right),
$$

onde $L^{i}\left(u_{k}\right)=\ln \left(p\left(u_{k}\right)\right)$ é a informação intrínseca, $\exp \left(\frac{-\left\|y_{k}^{s}-x_{k}^{s}\right\|^{2}}{N_{0}}\right)$ é a informação sistemática e

$$
L^{e}\left(u_{k}\right)=\ln \left(\sum_{\left(s^{\prime}, s\right)} \alpha_{k-1}\left(s^{\prime}\right) \cdot \beta_{k}(s) \cdot \gamma_{k}^{e}\left(s, s^{\prime}\right)\right),
$$

é a informação extrínseca.

Note que a informação extrínseca calculada na saída do primeiro decodificador, após ser entrelaçada, é usada como informação a priori na entrada do segundo decodificador. E de forma análoga, a informação extrínseca calculada na saída do segundo decodificador, após ser desentrelaçada, é usada como informação a priori na entrada do primeiro decodificador, de acordo com o processo iterativo.

Processo Iterativo: Considere inicialmente o primeiro decodificador componente na primeira iteração. Este decodificador recebe a seqüência de canal, $y^{1}$ (ver Figura 2), e produz uma estimativa da informação a posteriori $L_{11}\left(u_{k}\right)$ dos símbolos de dados $u_{k}$, onde $k \in$ $\{1, \cdots, N\}$ e $N$ é o comprimento da seqüência de informação. Note que nesta primeira iteração a informação a priori que o primeiro decodificador componente recebe é $\ln \left(p\left(u_{k}=\theta\right)\right)=\ln (1 / M)$.

$\mathrm{O}$ segundo decodificador componente recebe a seqüência de canal $y^{2}$ junto com a informação extrínseca entrelaçada do primeiro decodificador componente e fornece uma estimativa da informação a posteriori $L_{12}\left(u_{k}\right)$, dos símbolos de dado $u_{k}$. Esta informação extrínseca (usada no segundo decodificador) é calculada através da expressão (9) na saída do primeiro decodificador componente.

$\mathrm{Na}$ segunda iteração, o primeiro decodificador componente novamente processa sua seqüência recebida de canal $y^{1}$, mas dessa vez, ele também possui a informação a priori $L^{i}\left(u_{k}\right)$.
Esta informação a priori é fornecida pela porção extrínseca da informação a posteriori $L_{12}\left(u_{k}\right)$, calculada pelo segundo decodificador componente, na primeira iteração. Portanto, este decodificador pode produzir uma melhor informação $a$ posteriori $L_{21}\left(u_{k}\right)$, na saída do primeiro decodificador componente, na segunda iteração.

Este processo iterativo continua e, a cada iteração, em média a taxa de erro de bit diminui para uma mesma relação sinal ruído - SNR (signal-to-noise ratio).

\section{REsultados}

Neste artigo, fixamos comprimento da seqüência de informação em $N=2000$ bits, a taxa de codificação turbo em $1 / 2$, o número de iterações em 8 e a energia (raio) da constelação $M$-PSK em 1, para cada sistema M-ário simulado. $O$ desempenho destes sistemas serão analisados com relação a complexidade de decodificação, a capacidade de correção de erro, a cardinalidade do alfabeto e o mapeamento de bits para símbolos. Note que cada sistema $M$-ário, $M>2$, processa $2000 / \log _{2} M$ símbolos e a probabilidade de erro de símbolo é dividida por $\log _{2} M$ para dar origem a probabilidade de erro de bit.

\section{A. Esquema Turbo Binário com Modulação B-PSK}

Como já existem resultados na literatura ([1], [2], [4] e [5]) sobre o sistema turbo binário, apresentamos nesta seção os resultados simulados que serão usados como referência para efeito de comparação com os resultados dos sistemas turbo ternário, quaternário e 5-ário, apresentados nas próximas seções.

A Figura 3 apresenta as curvas da probabilidade $P_{e}(b)$ de erro de bit média versus $E_{b} / N_{0}$ para o esquema turbo binários que possui codificadores $R S C$ com $g(D)=\langle 1101 \mid 1111\rangle$ (treliça com $2^{3}=8$ estados) e $g(D)=\langle 11001 \mid 11011\rangle$ (treliça com $2^{4}=16$ estados).

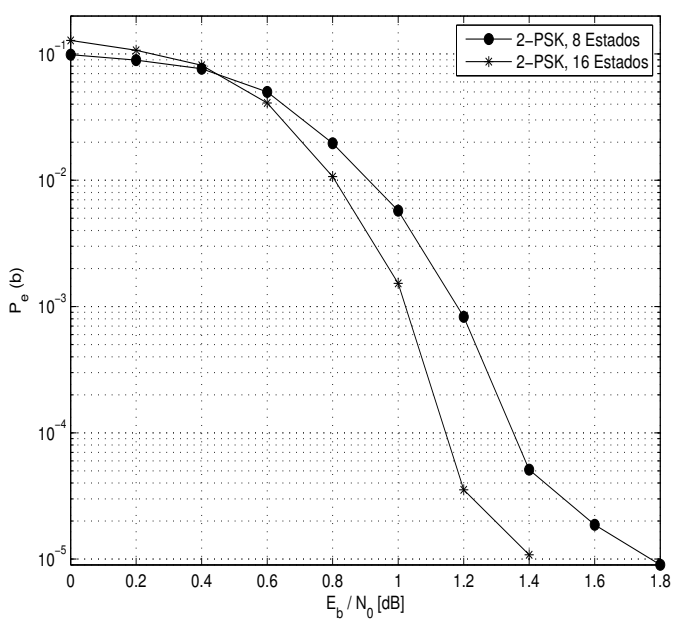

Fig. 3. Desempenho dos códigos $R S C$ binários.

A curva de desempenho, em termos de probabilidade de erro de bit média versus $E_{b} / N_{0}$, referente ao codificador cuja treliça possui 16 estados, apresenta melhor desempenho do que 
a referente ao codificador de 8 estados, para $E_{b} / N_{0}>0.5 \mathrm{~dB}$. Por outro lado, o tempo gasto pela decodificação no esquema referente a 16 estados é de 69.35 segundos e o tempo do esquema de 8 estados é de 37.56 segundos.

A Tabela I mostra a matriz geradora dos codificadores binários que possuem maiores distâncias $D_{\text {free }}$ e $D_{\text {efet }}[10]$, [8]. Observe que a distância euclidiana mínima do código turbo, $D_{\text {free }}$, é formada pelo peso euclidiano da seqüência de paridade do codificador $R S C-1$ mais o peso euclidiano da sequiência de paridade do codificador $R S C$-2 mais o peso euclidiano $W_{\text {free }}$, da seqüências de informação associada a $D_{\text {free }}$, enquanto que, a distância euclidiana efetiva $D_{\text {efet }}$, é a menor distância euclidiana associada ao peso efetivo $W_{\text {efet }}$, onde $W_{\text {efet }}$ é o peso da sequiências de informação não nula e de menor peso euclidiano, na qual a treliça do codificador $R S C$ tem [0 0$]$ como estado inicial e final. Note que $g(D)=\left[1 \frac{g_{2}(D)}{g_{1}(D)}\right]=\left\langle g_{1}(D) \mid g_{2}(D)\right\rangle=\left\langle a_{n} \cdots a_{0} \mid b_{n} \cdots b_{0}\right\rangle$, onde $a_{i}$ e $b_{i}$ são os coeficientes dos polinômios $g_{1}(D)$ e $g_{2}(D)$, respectivamente.

\begin{tabular}{||c|c|c|c|c|c||}
\hline Estados & $g(D)$ & $D_{\text {efet }}$ & $W_{\text {efet }}$ & $D_{\text {free }}$ & $W_{\text {free }}$ \\
\hline \hline 4 & $\langle 111 \mid 101\rangle$ & 20 & 4 & 14 & 6 \\
\hline 8 & $\langle 110|| 1111\rangle$ & 28 & 4 & 16 & 8 \\
\hline 16 & $\langle 11001 \mid 11011\rangle$ & 22 & 6 & 18 & 10 \\
\hline
\end{tabular}

TABELA I: Codificadores binários.

\section{B. Esquema Turbo Ternário com Modulação 3-PSK}

Nesta seção analisamos o desempenho e a complexidade do esquema turbo ternário com relação ao esquema turbo binário.

A Figura 4 apresenta as curvas da taxa de erro de bit versus $E_{b} / N_{0}$ para o esquema turbo ternário que possui codificadores $R S C$ com $g(D)=\langle 111 \mid 102\rangle$ (treliça com 9 estados) e $g(D)=\langle 1112 \mid 2122\rangle$ (treliça com 27 estados), comprimento da seqüência de informação, $N=1261$ símbolos .

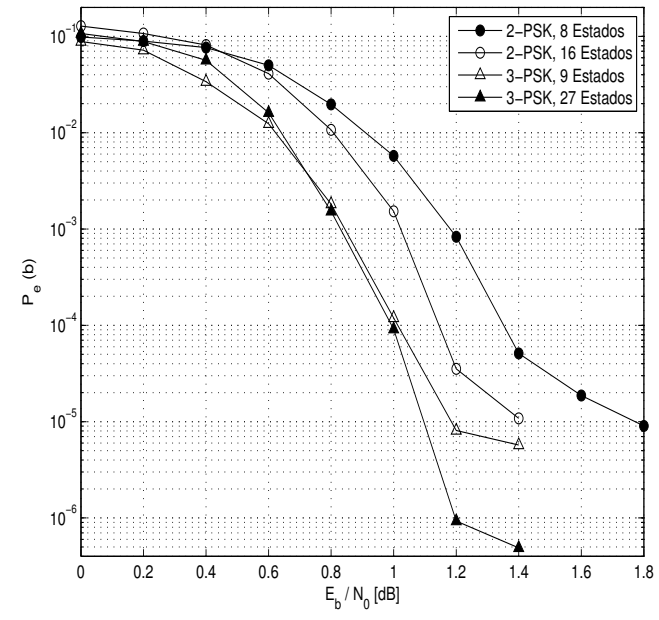

Fig. 4. Desempenho dos códigos turbo binários e ternários.

A curva de desempenho, em termos de probabilidade de erro de bit média versus $E_{b} / N_{0}$, referente ao codificador cuja treliça possui 27 estados, apresenta melhor desempenho do que a curva do codificador de 9 estados, para $E_{b} / N_{0}>0.7 \mathrm{~dB}$.
Além disso, para $E_{b} / N_{0} \geq 1.2 \mathrm{~dB}$ a probabilidade de erro de bit do codificador de 27 estados é pelo menos 1 ordem de grandeza menor que a probabilidade do codificador de 9 estados. Por outro lado, o tempo gasto na decodificação do esquema de 27 estados, é 2.19 vezes maior do que o tempo no esquema de 9 estados.

Logo, o esquema ternário com 9 estados tem bom desempenho e baixa complexidade de decodificação. Já o esquema ternário com 27 estados tem maior complexidade de decodificação e melhor desempenho que o esquema referente a 9 estados. Note também o erro floor mais baixo no esquema com 27 estados.

A Figura 4 também mostra o desempenho dos esquemas binário e ternário juntos. $\mathrm{O}$ esquema ternário com 9 estados, tem ganhos ${ }^{1}$ maiores do que $0.3 d B$ e $0.2 d B$, para $p_{e}(b)<$ $10^{-2}$, quando comparado com esquemas binários com $8 \mathrm{e}$ 16 estados, respectivamente. Além disso, o tempo gasto na decodificação do esquema ternário com 9 estados é 1.19 e 2.33 vezes menor que o tempo dos esquemas binários com 8 e 16 estados, respectivamente. Note que o sistema ternário processa $N=1261$ símbolos em vez de $N=2000$ bits do sistema binário.

Como o número de estados de cada codificador influenciam tanto no desempenho quanto na complexidade do sistema turbo, a comparação do sistema binário com o ternário não é muito justa, mas indica que os codificadores ternários proporcionam melhor desempenho.

Logo, o sistema ternário com modulação 3-PSK, apresenta melhor desempenho, menor complexidade de decodificação e ocupa menos largura de faixa que sistema binário com modulação 2-PSK. Ou seja, este sistema ternário transporta 1.58 vezes mais informação do que o sistema binário, tornando-se assim mais eficiente quando aplicado a sistemas de comunicação que possuam largura de faixa limitada. Todavia, este sistema não possui um perfeito mapeamento da fonte binária para os símbolos ternários.

A Tabela II mostra a matriz geradora dos codificadores ternários que possuem maiores distâncias $D_{\text {free }}$ e $D_{\text {efet }}$.

\begin{tabular}{||c|c|c|c|c|c||}
\hline Estados & $g(D)$ & $D_{\text {efet }}$ & $W_{\text {efet }}$ & $D_{\text {free }}$ & $W_{\text {free }}$ \\
\hline \hline 9 & $\langle 111 \mid 102\rangle$ & 20.78 & 3.46 & 12.12 & 5.19 \\
\hline 9 & $\langle 122 \mid 202\rangle$ & 20.78 & 3.46 & 13.85 & 6.92 \\
\hline 27 & $\langle 1112 \mid 2122\rangle$ & 24.24 & 3.46 & 15.58 & 5.19 \\
\hline
\end{tabular}

TABELA II: Codificadores ternários.

\section{Esquema Turbo Quaternário com Modulação 4-PSK}

A Figura 5 apresenta as curvas da taxa de erro de bit versus $E_{b} / N_{0}$ para o esquema turbo quaternário que possui codificador $R S C$ com $g(D)=\langle 113 \mid 212\rangle$ (treliça com 16 estados), comprimento da sequiência de informação, $N=1000$ símbolos.

O codificador quaternário apresenta desempenho equivalente ao desempenho do codificador binário com 16 estados e o esquema turbo quaternário processa $N=1000$ símbolos em vez de $N=2000$ bits como o sistema binário.

\footnotetext{
${ }^{1}$ Denotamos como ganho o valor absoluto da diferença entre as duas razões $E_{b} / N_{0}(1)$ e $E_{b} / N_{0}(2)$, calculada para a mesma taxa de erro de bit.
} 
Logo, o esquema quaternário com modulação 4-PSK gasta metade do tempo de decodificação em relação ao esquema binário com modulação 2-PSK. Ou seja este esquema quaternário processa o dobro da quantidade de informação do esquema binário com o mesmo tempo de decodificação.

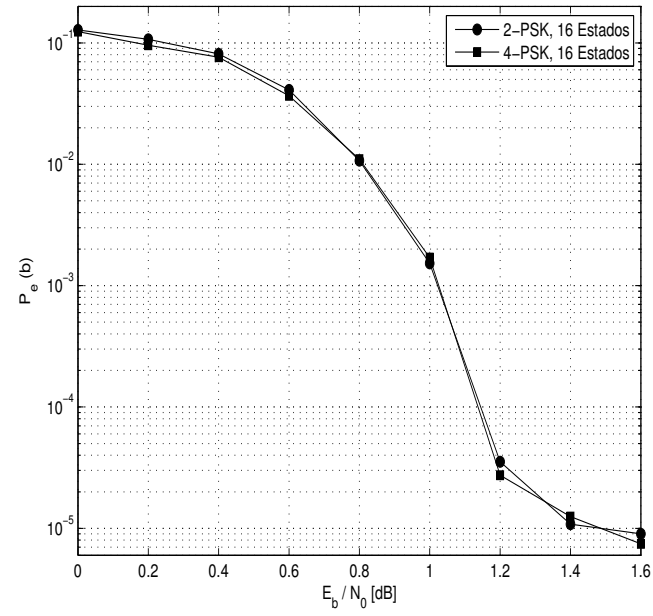

Fig. 5. Desempenho dos códigos turbo binários e quaternários.

A Tabela III mostra a matriz geradora dos codificadores quaternários que possuem maiores distâncias $D_{\text {free }}$ e $D_{\text {efet }}$.

\begin{tabular}{||c|c|c|c|c|c||}
\hline Estados & $g(D)$ & $D_{\text {efet }}$ & $W_{\text {efet }}$ & $D_{\text {free }}$ & $W_{\text {free }}$ \\
\hline \hline 16 & $\langle 111 \mid 232\rangle$ & 26.14 & 2.82 & 10.0 & 6 \\
\hline 16 & $\langle 113 \mid 212\rangle$ & 19.31 & 2.82 & 10.0 & 6 \\
\hline 16 & $\langle 133 \mid 312\rangle$ & 21.31 & 2.82 & 11.89 & 6.24 \\
\hline
\end{tabular}

TABELA III: Codificadores quaternários.

\section{Esquema Turbo 5-ário com Modulação 5-PSK}

Nesta seção analisamos o desempenho, a complexidade, o mapeamento de fonte e a largura de banda do esquema 5-ário com relação ao esquema binário.

A Figura 6 apresenta a curva da taxa de erro de bit versus $E_{b} / N_{0}$ para o esquema turbo 5 -ário que possui codificador $R S C$ com $g(D)=\langle 111 \mid 443\rangle$ (treliça com 25 estados), comprimento da sequiência de informação, $N=861$ símbolos.

A curva referente ao codificador 5-ário encontra-se acima da curva do codificador binário com 16 estados. Note ainda que o esquema 5-ário, realiza o processamento com $N=861$ símbolos, com 25 estados na treliça e com 5 transições associadas a cada estado, enquanto que o esquema binário, realiza o processamento com $N=2000$ bits, com 16 estados na treliça e com 2 transições associadas a cada estado. O tempo gasto na decodificação do esquema 5 -ário é 1.6 vezes menor, e a quantidade de informação transmitida é 2.3 vezes maior do que no esquema binário. Portanto, para um sistema que possua largura de faixa limitada, o esquema 5-ário é mais vantajoso que o esquema binário.

Codificadores $M$-ários que possuem 2 ou mais memórias apresentam melhor capacidade de codificação e melhor desempenho que o binário. Por outro lado, se o tamanho do alfabeto M-ário é maior do que 7, o sistema turbo torna-se impraticável devido a complexidade de decodificação, para um codificador com 2 ou mais memórias. Por exemplo, um codificador 8-ário com 2 memórias possui $8^{2}=64$ estados e com 3 memórias possui $8^{3}=512$ estados.

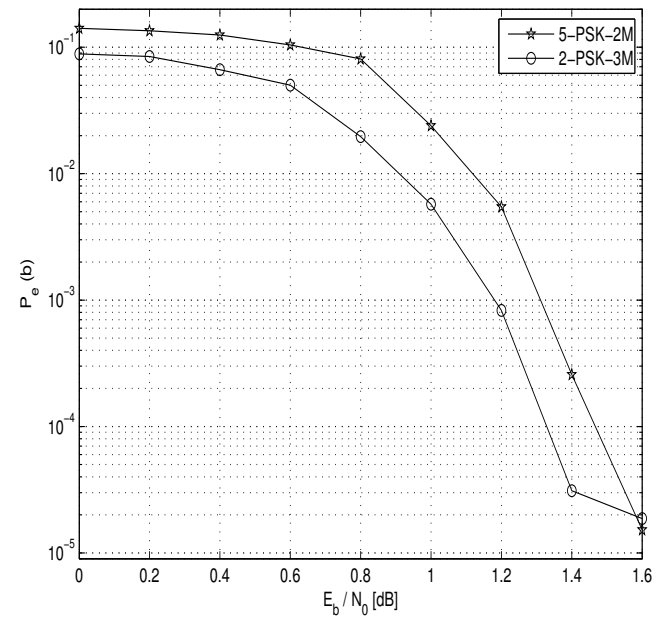

Fig. 6. Desempenho dos códigos turbo binários e 5-ários.

A Tabela IV mostra a matriz geradora dos codificadores 5-ários que possuem maiores distâncias $D_{\text {free }}$ e $D_{\text {efet }}$.

\begin{tabular}{||c|c|c|c|c|c||}
\hline Estados & $g(D)$ & $D_{\text {efet }}$ & $W_{\text {efet }}$ & $D_{\text {free }}$ & $W_{\text {free }}$ \\
\hline \hline 25 & $\langle 111 \mid 213\rangle$ & 21.54 & 3.07 & 12.31 & 6.16 \\
\hline 25 & $\langle 111 \mid 443\rangle$ & 17.73 & 3.07 & 12.03 & 3.52 \\
\hline 25 & $\langle 144 \mid 242\rangle$ & 17.56 & 3.35 & 13.20 & 3.82 \\
\hline
\end{tabular}

TABELA IV: Codificadores 5-ários.

Verificaremos agora o desempenho do sistema turbo $M$-ário com relação a variação do número de iterações, do comprimento da sequêencia de informação e do número de estados da treliça referente ao codificador $R S C$.

A Figura 7 mostra que as curvas referentes a $8^{\mathrm{a}}, 7^{\mathrm{a}}, 6^{\mathrm{a}}, 5^{\mathrm{a}}$, $4^{\mathrm{a}}, 3^{\mathrm{a}}, 2^{\mathrm{a}}$ e $1^{\mathrm{a}}$ iteração alcançam a probabilidade de erro de bit de $10^{-5}$ nas taxas de $1.19,1.20,1.28,1.38,1.49,1.79,2.40 \mathrm{e}$ $4.20 \mathrm{~dB}$, com tempos de decodificação de $21.71,19.38,17.00$, $14.70,12.33,9.95,7.61$ e 5.25 segundos, respectivamente.

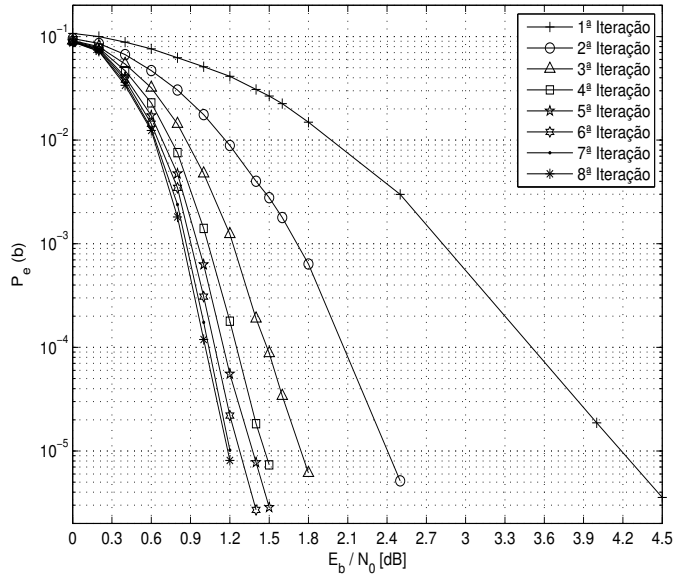

Fig. 7. Processo iterativo turbo ternário. 
Note que existe um determinado valor para o número de iterações tal que, se aumentarmos este valor, aumentamos em consequiência disto o tempo gasto na decodificação, mas não obtemos um ganho de codificação adicional significativo para o esquema turbo.

A Figura 8 mostra que as curvas na $8^{\mathrm{a}}$ iteração referentes aos comprimentos das sequiências de informação de $N=8000$, $N=4000, N=2000, N=1000$ e $N=500$ bits, alcançam a probabilidade de erro de bit de $10^{-5}$ nas taxas de $0.80,0.99$, $1.19,1.54$ e $2.00 \mathrm{~dB}$, com tempos de decodificação de 141.70, 50.93, 21.91, 10.02 e 4.89 segundos, respectivamente.

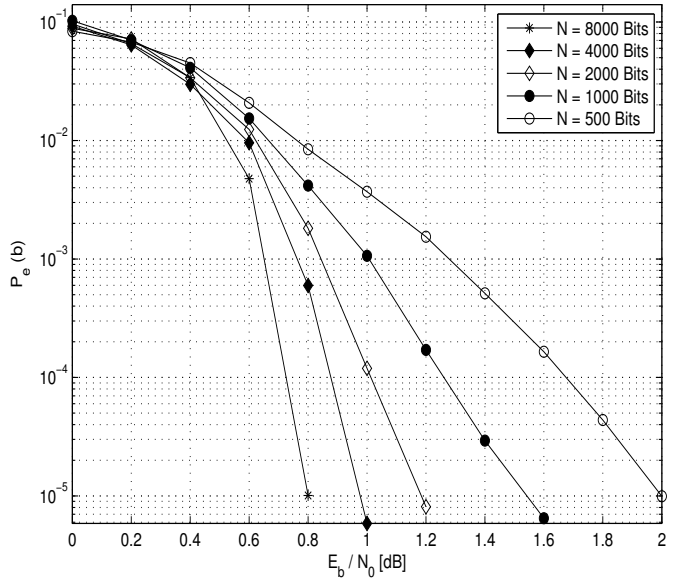

Fig. 8. Variação do comprimento da seqüência de informação do sistema ternário.

Note que existe um determinado valor para o comprimento da seqüência de informação que, a partir deste valor, se aumentarmos o comprimento da seqüência de informação, aumentamos em consequiência disto o tempo gasto na decodificação, mas o ganho de codificação obtido com esse aumento é muito pequeno.

A Figura 9 mostra que as curvas na $8^{\mathrm{a}}$ iteração do esquema binário referentes a 4,8 e 16 estados, alcançam a probabilidade de erro de bit de $2 \times 10^{-5}$ nas taxas de $1.3,1.6$ e $1.9 \mathrm{~dB}$, com tempos de decodificação de $69.35,37.56$ e 21.00 segundos, respectivamente, e que as curvas da $8^{\mathrm{a}}$ iteração do esquema
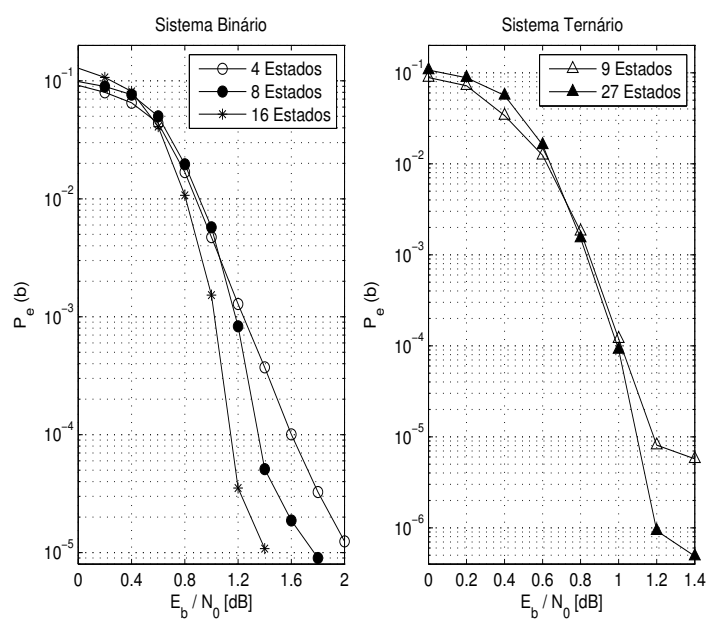

Fig. 9. Variação do número de estados. ternário referentes a 9 e 27 estados, alcançam a probabilidade de erro de bit de $6 \times 10^{-6}$ nas taxas de 1.4 e $1.11 \mathrm{~dB}$, com tempos de decodificação de 23.21 e 55.02 segundos, respectivamente. Note que existe um determinado valor para o número de estados da treliça do codificador $R S C$, que, a partir deste valor, o tempo gasto na decodificação é grande tornando assim o algoritmo de decodificação iterativo impraticável.

\section{CONCLUSÃO}

Nesta seção analisamos as curvas da taxa de erro de bit versus $E_{b} / N_{0}$ referente as simulações realizadas para os códigos turbo multiníveis definidos sobre os anéis e campos de inteiros módulo- $M$.

No sistema $M$-ário definido sobre o anel de inteiros $\mathbb{Z}_{M}$, existe um número reduzido de polinômios para a matriz geradora do codificador $R S C$, pois nem todo elemento do anel $\mathbb{Z}_{M}$ possui inverso multiplicativo. Por outro lado, alguns anéis possuem um perfeito mapeamento de fonte quando comparado com o sistema binário, como os anéis $\mathbb{Z}_{4}, \mathbb{Z}_{8}, \mathbb{Z}_{16}$ e etc.

No sistema $M$-ário definido sobre o campo $\mathbb{Z}_{M}$, existe um número bem maior de polinômios para a matriz geradora do codificador $R S C$, mas não existe um perfeito mapeamento da fonte binária nos símbolos de $\mathbb{Z}_{M}$.

As curvas simuladas mostram que os códigos turbo multiníveis apresentam bom desempenho com baixa relação sinal ruído e, em geral, quanto maior o tamanho do alfabeto maior será a eficiência do sistema turbo $M$-ário, pois 1 símbolo transmitido corresponde a $\log _{2} M$ bits transmitidos no sistema $M$-ário. Além disso, para cada sistema turbo $M$-ário existe uma faixa de valores para os parâmetros (tamanho do alfabeto, comprimento da seqüência de informação, número de iterações, taxa de codificação e codificador $R S C$ ) para os quais o sistema turbo apresenta melhor desempenho.

Os resultados simulados mostram que o esquema turbo ternário, que possuem codificadores componentes com 9 estados, apresentam melhor desempenho com menor complexidade de decodificação que os demais esquemas turbo M-ários analisados.

\section{REFERÊNCIAS}

[1] C. Berrou, A. Glavieux and P. Thitimajshima, "Near Shannon Limit Error-Correcting Coding and Decoding: Turbo Codes," Proc. Int. Conf. Comm., pp. 1064-1070, May 1993.

[2] J. Hagenauer, E. Offer, and L.Papke, "Iterative decoding of binary block and convolutional codes," IEEE Trans. Inform. Theory, vol. 42, pp. 429445, Mar. 1996.

[3] G. Ungerboeck, "Channel coding with multilevel/phase signals," IEEE Trans. Inform. Theory, pp. 55-67, Jan. 1982

[4] P. Robertson, "Illuminating the structure of code and decoder of parallel concatenated recursive systematic (turbo) codes," Proc. Globecom. pp. 1298-1303, 1994

[5] W. E. Ryan, “A Turbo Code Tutorial,'Proc. IEEE Globecom'98, 1998.

[6] M. F. Atiyah and I. G. MacDonald, "Commutative Algebra," Reading, MA: Addison - Wesley, 1969.

[7] Rolf Johannesson, "Some Structural Properties of Convolutional Codes over Rings," IEEE Trans. Inform. Theory, pp. 839-845, Mar. 1998.

[8] Chris Heegard and Stephen B. Wicker, "Turbo Coding," Kluwer Academic Publishers pp. 10-63.

[9] F. Fagnini and S. Zampieri, "System-Theoretic Properties of Convolutional Codes Over Rings," IEEE Trans. Inform. Theory, vol. 47, $\mathrm{N}^{o} .6$, Sept. 2001, pp. 2256-2274.

[10] S. Benedetto and G. Montorsi, "Design of Parallel Concatenated Convolutional Codes," IEEE Trans. Comm., vol. 44, N ${ }^{o} .5$, May. 1996, pp. 591-600. 